\title{
The effect of the presence of the patient on the particles dose estimations in high energy linear accelerator mazes
}

\author{
Jillali Ghassoun \\ Department of Physics, Faculty of Sciences Semlalia, Cadi Ayyad University, Marrakech, Morocco \\ [Presented at the $4^{\text {th }}$ African Regional IRPA congress (AFRIRPA04), which was held from September 13-17, 2014 in Rabat, Morocco. This paper \\ was reviewed and accepted by the scientific committee of the $4^{\text {th }}$ African Regional IRPA congress]
}

\section{Conference Proceeding}

\begin{abstract}
Purpose: Medical accelerators operating above $10 \mathrm{MV}$ are a source of undesirable neutron radiations which contaminate the therapeutic photon beam. These photo-neutrons which contaminate the therapeutic beam can also generate secondary gamma rays, via inelastic and capture reactions, which increase the undesirable dose to the patient body, the oncology staff and the general public. The purpose of the present work is to investigate, through Monte Carlo simulation, the effect of the presence of the patient on the neutron and gamma rays dose calculations. Methods: To illustrate this effect, the MCNP5 code was used to model a radiotherapy room of a medical linear accelerator operating at $18 \mathrm{MV}$ and to calculate the neutron and the secondary gamma ray energy spectra and the dose equivalent, at various points along the centerline of the maze, in the absence and presence of the patient. Results and Conclusion: The obtained results show a significant change in the neutron energy spectra in the presence of the patient especially in the thermal neutron energy region. The results also indicate that the presence of a patient does not affect the simulated neutron and gamma rays dose equivalents at the maze entrance for mazes greater than $3 \mathrm{~m}$ long. A significant change in dose equivalent calculated values was observed when the length of the maze is less than $3 \mathrm{~m}$.
\end{abstract}

Keywords: Radiotherapy; Accelerator; Neutron; Gamma; Phantom; Dose; MCNP; Maze

\section{Introduction}

Medical linear accelerators operating above $10 \mathrm{MV}$ produce photo-neutrons via the interaction of high-energy photons with high atomic number $(Z)$ materials. These photo-neutrons which contaminate the therapeutic beam can also generate secondary gamma rays, via inelastic and capture reactions, which increase the undesirable dose delivered to the patient, the oncology staff and the general public. It is therefore advisable to have an accurate evaluation of neutron dose produced in the radiotherapy room and in the surrounding areas.

In a previous work, the Monte Carlo method was used to model a radiotherapy room of a medical linear accelerator operating at $18 \mathrm{MV}$ and to evaluate the dose equivalent distributions of neutrons and secondary gamma rays in a liquid tissue-equivalent (TE) phantom and at various points inside the treatment room and along the maze. ${ }^{1,2}$

The purpose of the present work is to investigate, through Monte Carlo simulation, the effect of the presence of the patient on the neutron and gamma ray dose estimations. To illustrate this effect, the MCNP5 code was used to model radiotherapy room of a medical linear accelerator operating at $18 \mathrm{MV}$ and to calculate the neutron and the secondary gamma ray energy spectra and the dose equivalent, at various points along the centerline of the maze, when the patient is present and absent. The simulations were performed using the MCNP5 Monte Carlo Code (X-5 Monte Carlo Team, 2005). ${ }^{3}$ The neutron dose equivalents were determined by calculating neutron multi-energy particles fluxes and convolving this data with the NCRP-38 flux-to-dose conversion factors (NCRP, 1971). ${ }^{4}$ The photon dose equivalents were calculated using the conversion factors (ICRP, 1974)..$^{5}$ The thermal neutron region was defined to be below $0.5 \mathrm{eV}$, the epithermal neutron region is from $0.5 \mathrm{eV}$ to $10 \mathrm{keV}$ and the fast neutron region is over $10 \mathrm{keV}$. The MCNP solid-state $\mathrm{S}(\alpha, \beta)$ neutron scattering library (lwtr.01t) was used in order to improve the accuracy of low energy neutron transport calculations. The calculations were performed with a relative error lower than $1 \%$.

\section{Methods and Materials}

\section{Monte Carlo simulation}

The application of the Monte Carlo method in medical physics covers almost all topics, including radiation protection, diagnostic, radiotherapy and nuclear medicine. In this 
study, the Monte Carlo method was used to simulate a medical linear accelerator operating at $18 \mathrm{MV}$ (Saturne linac). Figure 1 shows the floor plan view of the simulated radiotherapy room. We assumed that all walls including the floor and the ceiling are made of ordinary concrete (NCRP, 1976). ${ }^{6}$ The distance from the floor to the ceiling was $2.7 \mathrm{~m}$.

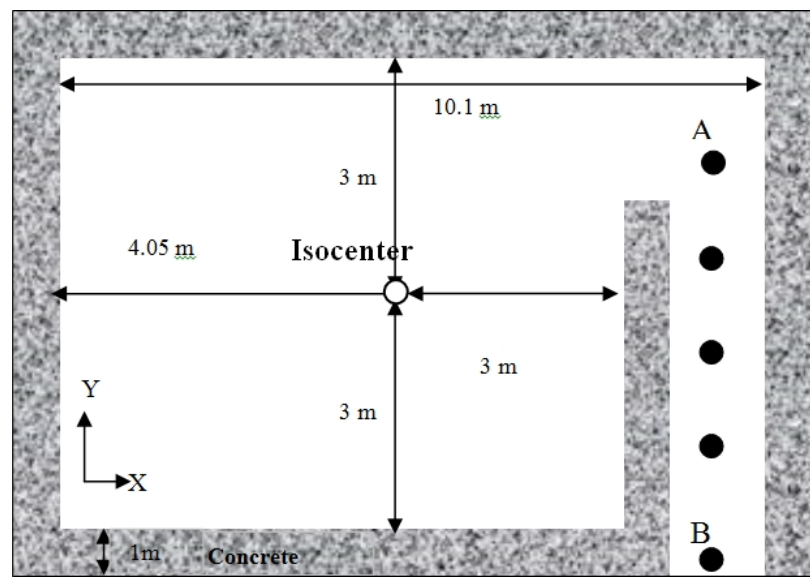

FIG. 1: Simulated radiotherapy room geometry for Monte Carlo calculations.

In order to study the effect of the presence of the patient on the neutron and gamma rays dose calculations, a $100 \times 30 \times$ $20 \mathrm{~cm}^{3}$ parallelepiped liquid tissue-equivalent (TE) phantom representing a patient body (ICRU, 1989) located at a $100 \mathrm{~cm}$ source-surface distance (SSD) is included in the model. ${ }^{7}$ The Monte Carlo N-Particle MCNP5 code was used to calculate the neutron and gamma rays energy spectra and dose equivalent at the maze inner entrance (point A in Figure 1) and at the maze entrance door (point B in Figure 1). All calculations were done for a field size of $10 \mathrm{~cm} \times 10 \mathrm{~cm}$. Neutron and gamma ray fluences were tallied in spherical cell tallies of 10 $\mathrm{cm}$ radius using the track length estimator (tally type $\mathrm{F} 4$ ).

In this simulation, the accelerator head was modeled as a 10 $\mathrm{cm}$ radius tungsten sphere around the source where neutrons are produced. The source is described as an isotropic point-like source with an energy spectrum given by Eq. (1) (Tosi et al., 1991): ${ }^{8}$

$\frac{\mathrm{dN}}{\mathrm{dE}_{\mathrm{n}}}=\frac{0.8929 \mathrm{E}_{\mathrm{n}}}{\mathrm{T}^{2}} \exp \left(-\mathrm{E}_{\mathrm{n}} / \mathrm{T}\right)+\frac{0.1071 \mathrm{Ln}\left(\mathrm{E}_{\max } /\left(\mathrm{E}_{\mathrm{n}}+7.34\right)\right)}{\int_{0}^{\mathrm{E}_{\max }-7.34} \operatorname{Ln}\left(\mathrm{E}_{\max } /\left(\mathrm{E}_{\mathrm{n}}+7.34\right)\right) \mathrm{dE}_{\mathrm{n}}}$

where, Emax is the maximum energy of the photons (in $\mathrm{MeV}$ ) and $\mathrm{T}$ is the nuclear temperature of the target material (in $\mathrm{MeV})$.

\section{Results and Discussions}

Figure 2 shows the neutron energy spectra calculated at the maze inner entrance (point A in Figure 1) with the presence and the absence of the patient. The fluence values are normalized per neutron emitted from the source. The neutron spectra at the maze inner entrance present two peaks: a thermal neutron peak below $1 \mathrm{eV}$ and a fast neutron peak from 0.1 to $10 \mathrm{MeV}$. We noticed that the presence of the patient substantially decreased the thermal neutron fluence. The epithermal neutron and fast neutron fluences are not affected by the presence of the patient. The change in the neutron spectra is a direct result of the absorption process of thermal neutrons in the patient's body.

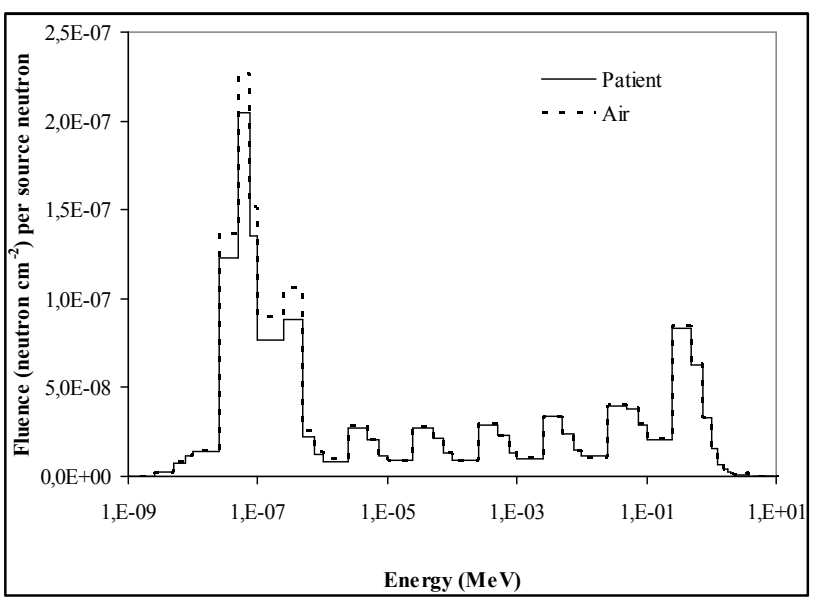

FIG. 2: The neutron energy spectra calculated at the maze inner entrance (point A in Figure 1) with the patient present and absent (air).

Figure 3 shows the neutron energy spectra calculated at the maze entrance (point B in Figure 1) in the absence and presence of the patient. We notice that at the maze entrance, the neutron fluences are very much reduced due to the maze. These results also show a significant change in the neutron energy spectra in the presence of the patient especially in the thermal energy region while the effect of the patient on epithermal and fast neutron fluences is not significant.

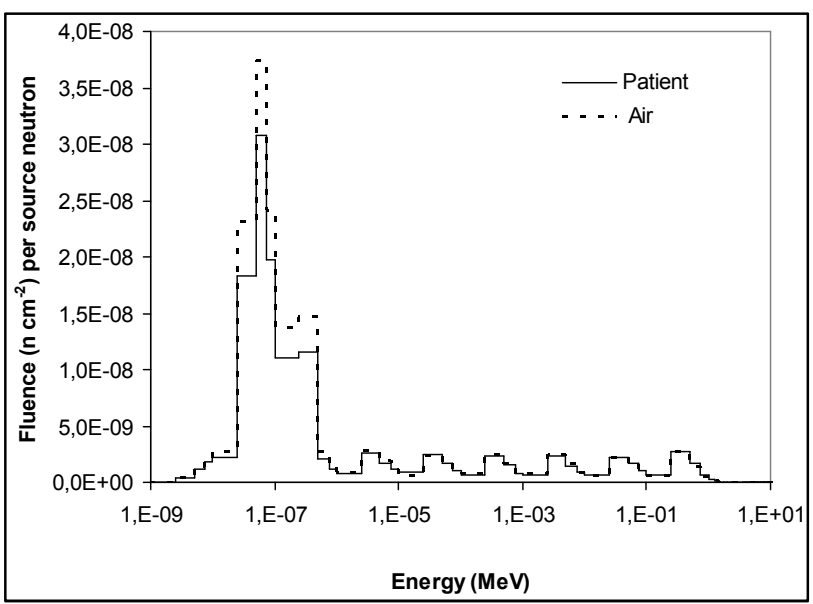

FIG. 3: The neutron energy spectra calculated at the maze entrance (point B in Figure 1) with the patient present or absent (in air). 
Figure 4 shows the neutron dose equivalents, in Sv per x-ray Gy at the isocenter, calculated at several points along the maze, extending from the maze inner entrance (point $A$ in Figure 1) to the vicinity of the maze entrance door (point $B$ in Figure 1) in the presence and the absence of the patient. The results indicate that the neutron dose equivalent decreases when moving towards the door. The results also demonstrate that the presence of the patient doesn't have a significant effect on the neutron dose equivalent estimations at the maze entrance for accelerator rooms with lengths greater than $3 \mathrm{~m}$. However, a significant change in neutron dose equivalent was observed for a short maze with a length less than $3 \mathrm{~m}$.

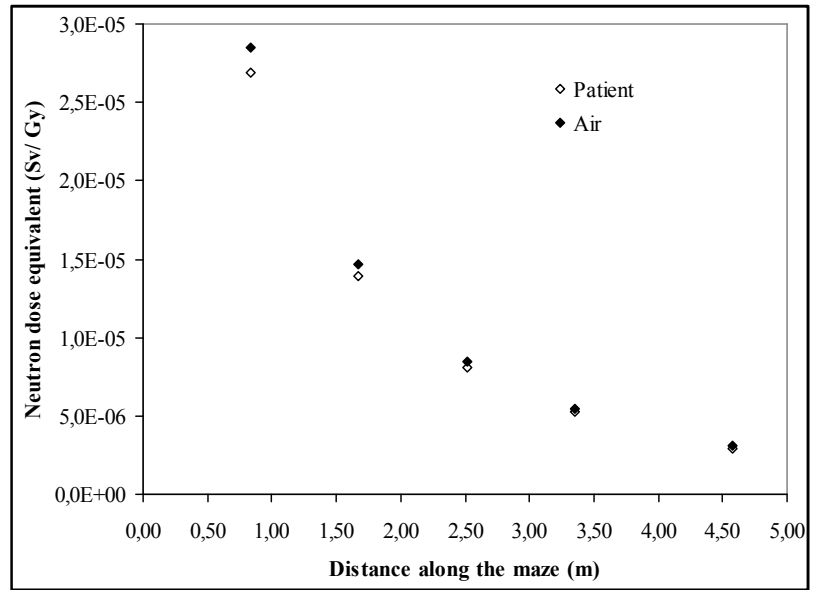

FIG. 4: The neutron dose equivalents, in Sv per x-ray Gy at the isocenter, calculated at several points in the maze with the patient present or absent.

Figure 5 provides a comparison between calculated secondary gamma-ray dose equivalents along the maze, in terms of Sv/Gy per $\mathrm{x}$-ray Gy at the isocenter, when the patient is present or absent. As it can be seen, the gamma-ray dose equivalent decreases with distance from the inner maze entrance (point A) to the maze entrance door (point B). A significant difference in gamma ray equivalent distributions through the maze is observed for maze of lengths less than $3 \mathrm{~m}$. This is not the case for mazes with lengths greater than $3 \mathrm{~m}$, where the two distributions are practically identical.

Figure 6 shows the behavior of the secondary gamma ray energy spectra obtained at the maze entrance with the patient present or absent. The capture of slow neutrons gives rise to several peaks. The pronounced peak at $2.223 \mathrm{MeV}$ is due to gamma rays generated by the neutron capture $H(n$, $\gamma) \mathrm{D}$ reaction with hydrogen:

$$
{ }^{1} \mathrm{H}+{ }^{1} \mathrm{n} \rightarrow{ }^{2} \mathrm{D}+\gamma(2.223 \mathrm{MeV})
$$

The other prominent peaks are attributed to calcium (1.95 $\mathrm{MeV}, 64 \mathrm{MeV}$ ), silicon (3.54 MeV, $4.94 \mathrm{MeV})$, iron (7.25 $\mathrm{MeV}$ ) and aluminum (7.75 MeV). The obtained results also show a significant change in the intensities of the gamma ray peaks in the presence of the patient as a direct result of the magnitude of the neutron capture process.

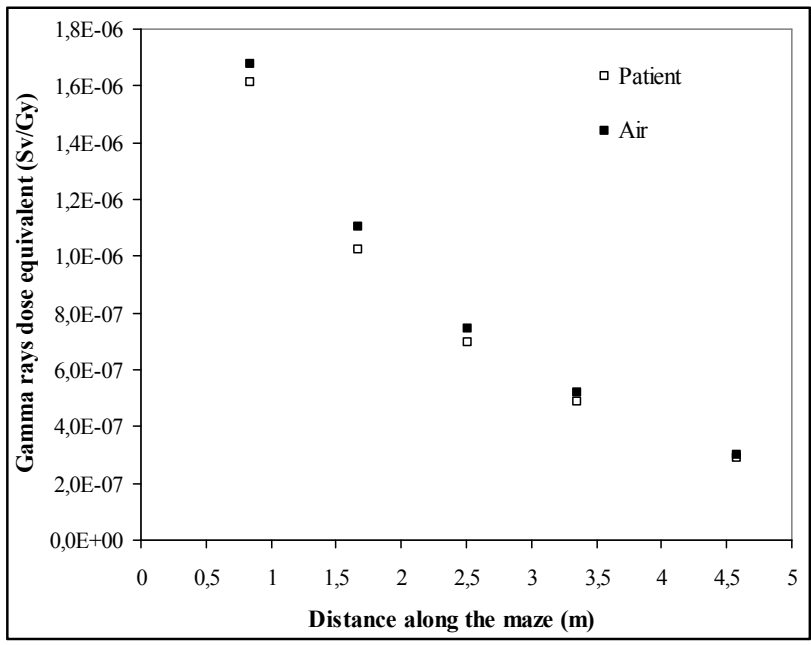

FIG. 5: Secondary gamma rays dose equivalent calculated at several points in the maze with the patient present or absent.

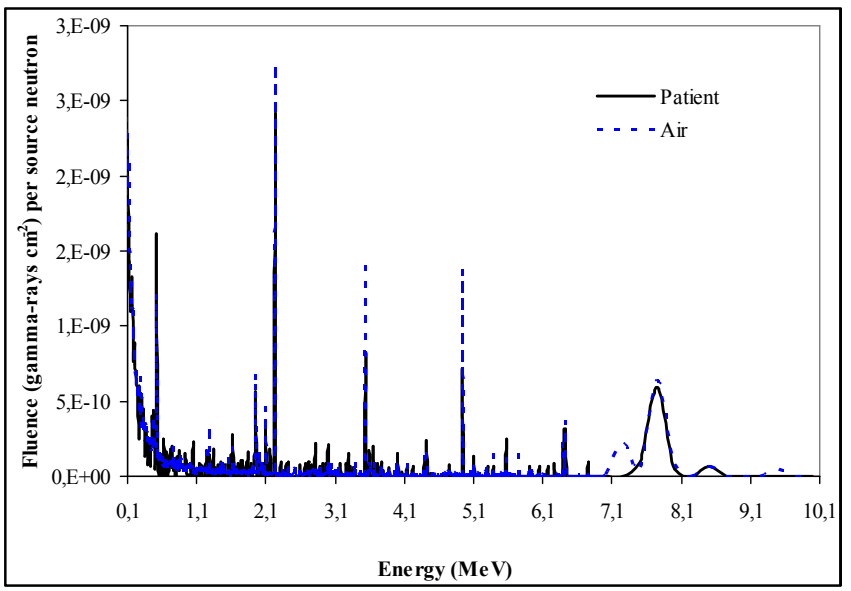

FIG. 6: The gamma ray energy spectra calculated at the maze entrance with the patient present or absent.

\section{Conclusion}

In this study, we have investigated, through Monte Carlo simulation, the effect of the presence of the patient on the neutron and gamma ray dose calculations in high-energy linear accelerator mazes. To illustrate this effect, the MCNP5 code was used to model a radiotherapy room of a medical linear accelerator operating at $18 \mathrm{MV}$ and to calculate the neutron and the secondary gamma ray energy spectra and the dose equivalent, at various points along the centerline of the maze, with the patient present and absent. The obtained results show a significant change in the neutron energy spectra in the presence of the patient especially in the thermal energy region. The results also indicate that the presence of a patient does not affect the simulated neutron and gamma ray dose equivalents at the maze entrance for mazes greater than $3 \mathrm{~m}$ long. A significant change in the 
estimated dose equivalent values was observed when the length of the maze is less than $3 \mathrm{~m}$.

This study provides useful information on the radiation protection of the patient, the workers and general public. The simulated linear accelerator was a Saturne 20 (CGR) but the method described here could be easily extended to other medical accelerators as well.

\section{Conflict of interest}

The authors declare that they have no conflicts of interest. The authors alone are responsible for the content and writing of the paper.

\section{References}

1. Ghassoun J, Senhou N, Jehouani A. Neutron and photon doses in high energy radiotherapy facilities and evaluation of shielding performance by Monte Carlo method. Ann Nucl; Energy. 2011; 38: 2163-7.

2. Ghassoun J, Senhou N. The evaluation of neutron and gamma ray dose equivalent distributions in patients and the effectiveness of shield materials for high energy photons radiotherapy facilities. Appl Radiat Isot. 2012;70:620-4.

3. X-5 Monte Carlo Team, MCNP - A General Monte Carlo N-Particle Transport Code, Version 5. Los Alamos National Laboratory report LAUR- 03-1987 (April 2003; Revised October 3, 2005).

4. NCRP. Report No, 38 - Protection against neutron radiation. Bethesda, Maryland; NCRP 1987.

5. Conversion coefficients for use in radiological protection against external radiation. Adopted by the ICRP and ICRU in September 1995. Ann ICRP. 1996;26:1-205. Review. Erratum in: Ann ICRP 1997;27:163-4.

6. NCRP. Report No, 49 - Structural shielding design and evaluation for medical use of X-rays and gamma rays of energies up to $10 \mathrm{MeV}$. Bethesda, Maryland; NCRP 1976.

7. ICRU. Tissue Substitutes in Radiation Dosimetry and Measurement (Report 44). Bethesda, Maryland; Intl Commission on Radiation. 1989.

8. Tosi G, Torresin A, Agosteo S, et al. Neutron measurements around medical electron accelerators by active and passive detection techniques. Med Phys. 1991;18:54-60. 